\title{
Heuristics and CAD modelling: an examination of student behaviour during problem solving episodes within CAD modelling activities
}

\author{
Jeffrey Buckley $^{1}$ (D) Niall Seery ${ }^{1,2} \cdot$ Donal Canty $^{3}$
}

Accepted: 16 August 2017/Published online: 31 August 2017

(C) The Author(s) 2017. This article is an open access publication

\begin{abstract}
Design activities typically involve and culminate in the creation of models representative of new ideas and conceptions. The format is often dictated by the specific discipline, with ideas in design and technology education regularly being externalised through the use of computer aided design (CAD). This paper focusses on the realisation stage of a design process, specifically when conceptual ideas are being externalised through CAD. Acknowledging students as novices or quasi-experts with regards to their levels of technical expertise and recognising the limitations in the cognitive capacities of humans suggests merit in investigating problem solving strategies through the lens of heuristics. A comparative study was employed between two distinct CAD systems to examine students modelling behaviour. Considering the situational context of the problems encountered and the bounded rationality which the students are operating within, a number of insights are generated from the findings which are of importance from a pedagogical perspective within design and technology education.
\end{abstract}

Keywords Heuristics · Design behaviour - Design cognition · Modelling · Computer aided design $\cdot$ Problem solving

\section{Introduction}

Humans differ in the amount of intelligence ascribed to them (Raab and Gigerenzer 2005) and the knowledge afforded to a person allowing them to operate within a domain is contained within their relevant schema. When engaging with a problem or task, the

Jeffrey Buckley

jeffbuckley1992@gmail.com

1 KTH Royal Institute of Technology, Stockholm, Sweden

2 Athlone Institute of Technology, Co. Westmeath, Ireland

3 University of Limerick, Co. Limerick, Ireland 
knowledge contained within acquired schema can be recalled from the long-term memory into the working memory, with the comprehensiveness of the schema and the successful transfer of this knowledge between memory systems being significant determinants of a person's capacity for success. Humans have the constraint of a limited capacity in their working memory system which is suggested to be approximately seven chunks of information (Miller 1956) however it has since been suggested that this number may be as low as four (Cowan 2001). This limitation can prevent a person recalling necessary information while problem solving, however in many respects it is circumvented as "information can be brought back from long-term memory to working memory over indefinite periods of time" (Kirschner et al. 2006, p. 77). In certain circumstances, such as when problem solving, this limitation is significant as access may be required to more chunks of information at a given time than a person's working memory capacity permits. These circumstances are a differentiating characteristic between novices and experts, as having more comprehensively developed schema allows experts to hold more bits of information within each chunk (Chase and Simon 1973).

Students within design and technology education can arguably be described as novices in the domain because they haven't had the opportunity to acquire extensive schema pertinent to the discipline. At most, students could be defined as quasi-experts or "individuals with more experience in a given domain than novices, but who do not have a recognized standing as experts" (Kaufman et al. 2013, p. 332) because they are still in a developmental process of learning and acquiring pertinent schema. Therefore, students regularly engage with activities while not possessing all necessary associated knowledge. Kimbell $(2011$, p. 7) eloquently describes this scenario by referring to a student being in a state of "half-knowing" which suggests that the solution that students can strive for can only be optimal within their bounded rationality. To counteract this limitation, when a search for relevant cues of information is unsuccessful, students can exhibit heuristics to aid them in solving a problem (e.g. Gigerenzer and Goldstein 1996). Through employing appropriate heuristics, students can attain a solution which satisfies a problem despite not having access to all relevant information. As "observed behaviour reflects the underlying cognitive abilities of the individual" (Raab and Gigerenzer 2005, p. 188), examining the problem solving strategies of design and technology students through the lens of heuristics and behaviours is of interest to the discipline. These observations can provide insight into the influence that the nature of an activity can have on thinking and into levels of student attainment.

\section{Problem solving behaviours and learning}

The nature of the learning environment typical of design and technology education sees students regularly encountering a variety of types of problems (Williams et al. 2008). Design is one specific context which involves complex problem solving activity (Schütze et al. 2003). The encountered problems both surround and are encompassed within tasks that students engage in. Schoenfeld (1983, p. 41) defines a problem as "only a problem... if you don't know how to go about solving it". If a problem does not hold this characteristic then it is more accurately defined as a task or an exercise and under this definition it is clear that both a task and a problem can be sub-activities of each other within a learning activity.

Problems are critical within education as they support student inquiry into a subject affording the opportunity to acquire and develop schema. Through the process of engaging 
with the unknown, new information can be identified which can then be accommodated or assimilated into pre-existing schema. Piaget (1970) identifies this process as cognitive adaption, defining assimilation as "the integration of external elements into evolving or completed structures" (p. 706) and accommodation as "any modification of an assimilatory scheme or structure by the elements it assimilates" (p. 708). Given the complexity of human cognition, no person experiences this process the same as another. Two contributory factors underpinning these experiences are cognition and behaviour. Cognitively, the uniqueness of each person's pre-existing schema will impact on the nature of the assimilation and accommodation processes. Behaviourally, the judgements and decisions made during the problem solving episode will impact what occurs during it. Considering the previously described limitations of the human memory system, these behaviours can take the form of heuristics where actions are taken to reduce effort within an activity (Shah and Oppenheimer 2008).

\section{The role of heuristics in problem solving}

To exemplify the role of heuristics in human activity, it is worth considering the opposing process that can be taken which involves considering all relevant information to arrive at an 'optimal' solution. In judgement and decision making, the weighted additive rule is a complex algorithm for arriving at an optimal decision which aims to describe the process of attaining and considering all relevant information (Payne et al. 1993). It requires people to expend cognitive effort on five tasks which include:

1. Identifying all cues,

2. Recalling and storing cue values,

3. Assessing the weights of each cue,

4. Integrating information for all alternatives,

5. All alternatives should be compared, and then the alternative with the highest value should be stored (Shah and Oppenheimer 2008).

This algorithm is equally valid in the study of problem solving behaviours as when engaging with a problem a person will make numerous decisions regarding their approach, its implementation and evaluation. When posed with a problem while engaging in designerly activity, the complexity of the involved decisions increases due to the divergent nature of the activity and a selection process occurs where the person must choose one of many potential solutions to strive for. Within problem solving however there is always an unknown entity which must be negotiated (Schoenfeld 1983) whereas in decision making and judgement it is feasible for all pertinent information to be known.

Operationalising the weighted additive rule within the context of solving a problem would impose significant cognitive load (Sweller 1988) thus reducing the cognitive resources available to learn during the problem. As previously discussed people have a limited processing capacity and, in addition to this, problems also occur within specific environments which must be negotiated. Amalgamating these two factors situates a person solving a problem within a bounded rationality (Simon 1955, 1956), an environment unique to the individual describing their relationship to the problem based on the interplay between available cognitive resources and the task environment. As the cognitive load increases as posed by the problem within the specific context, heuristics can be employed to reduce mental effort, allowing for optimisation under situational constraints (Sargent 1993). Therefore, from a pedagogical perspective, heuristics have the capacity to support learning by giving access to cognitive resources which can be allocated to learning and 
sense-making processes. Their employment however can also have negative implications if information which is at the core of the learning activity is not sought by the student. Alternatively, actions can be taken which alleviate the need to seek pertinent information in the first place.

\section{Employing heuristics: the adaptive toolbox}

When considering the employment of heuristics, it is important to consider human activity and intelligence from a broader perspective to rationalise why they are important. Two of the prominent schools of thought on human intelligence include viewing intelligence as predominantly cognitive or internal activity and as predominantly behaviouristic or external activity. In both views human intelligence is conceptualised as a series of individual processes which culminate into a holistic structure. The former is the concept that human intelligence is constructed of a number of cognitive factors (e.g. Schneider and McGrew 2012), a construct whose origins can be traced to the early work of Galton (1880) and Spearman (1904). When studying intelligence, this conceptual framework is limited because it does not describe how cognition translates into human behaviour (Sternberg et al. 2001). The second school of thought, which is behaviouristic, is that of fast and frugal heuristics as a model of intelligence consisting as parts of a larger system known as the adaptive toolbox (Raab and Gigerenzer 2005). The heuristics are fast as they can help solve problems quickly and frugal as they require little information (Gigerenzer 2004). The adaptive toolbox is designed to achieve proximal goals and consists of a number of tools such as search rules, stopping rules and decision rules (Gigerenzer 2001). In the context of problem solving, search rules describe the process of searching for information, stop rules denote strategies by which this search is stopped and decision rules describe how a decision or inference is made subsequent to stopping the search. These rules are exhibited through the employment of heuristics. Gigerenzer (2008) presents a series of heuristics likely to be included in the adaptive toolbox which people can use adaptively either with or without awareness. The selection process involved in choosing a heuristic is guided by individual reinforcement learning, social learning and evolutionary training (Hutchinson and Gigerenzer 2005). What is critical within this selection process is that the heuristics within the adaptive toolbox are domain specific rather than domain general (Gigerenzer 2001). Therefore the situational context that the heuristics are being selected within is of critical importance and the ecological rationality of the heuristic must be recognised.

\section{Ecological rationality in the context of CAD modelling}

Ecological rationality concerns the compatibility between strategy and environment. Heuristics that are matched within their environments allow "agents to be ecologically rationale, making adaptive decisions that combine accuracy with speed and frugality" (Gigerenzer 2001, p. 47) Therefore when studying heuristics and behaviours within education, it is imperative to understand the ecological environment the student is operating within. This environment does not necessarily need to be the physical space embodied by the student. It can also be a virtual space which their actions manifest within such as a computer aided design (CAD) modelling environment.

Design tasks within design and technology education are typically divergent and therefore "may have multiple solutions or no solution at all, nor is there a guaranteed procedure to reach that solution" (Schraw et al. 1995, p. 523-524). Therefore when CAD is being used as a medium for externalising ideas, this activity becomes goal orientated as 
multiple ideas are created to support interaction between the student and their conceptions and students' goals involve generating models to support this dialogue. Considering the relative complexity of $\mathrm{CAD}$, it is conceivable that its utilisation would be positioned within educational agendas both to afford students the capacity to develop the more speculative competencies broadly inherent within designerly thinking with the more critical competencies of strategic CAD modelling. This dichotomy between designerly capacities and discipline specific knowledge, such as strategic CAD modelling knowledge, is of substantial importance because if either is underdeveloped in their amalgamation the weaker can reduce a student's capacity to enact the other. For example, a distinct lack of disciplined knowledge could hinder the design process by impeding the critical refinement of a speculative idea. Similarly, in a designerly context, a student may have the capacity to utilise a modelling tool, but be unable to identify it as an appropriate tool at a given time or be unable to determine how it should be used.

Therefore, in order to create CAD models of their conceptual ideas, students will need to have attained the relative level of capacity to operate the CAD system. This level of capacity is dictated by a level of strategic knowledge and an understanding of design intent. It is widely regarded that expertise in CAD modelling is differentiated by strategic modelling knowledge rather than command knowledge (Bhavnani et al. 1993; Lang et al. 1991; Rodriguez et al. 1998). However it is conceivable that this level of knowledge may not be attained by students because they can be "so busy learning the commands that little time is available for acquiring other kinds of information such as procedural [strategic] knowledge" (Lang et al. 1991, p. 257; Chester 2007, p. 24-25). A consequence of not attaining the required level of strategic knowledge is that "without proper forethought, construction of a solid model can reach a critical stage where parametric manipulation is no longer possible" (Rodriguez et al. 1998, p. 1) which may eliminate the efficacy of the model itself. Coupled with the need to externalise a conceptual idea through CAD, students must also consider the design intent of the model. Design intent is a similar concept to strategic knowledge (Chester 2007) in that it refers to "strategies for incorporating maximum design flexibility and minimising design failure" (Rynne et al. 2003, p. 2). Therefore, taking cognisance of the volume of discipline specific knowledge associated with CAD modelling, its implementation as a pedagogical tool in a designerly context merits investigation to ensure an appropriate synthesis between learning objectives and student behaviours stemming from their associated levels of technical and designerly competencies.

\section{Hypothesis}

The juxtaposition of divergent design tasks with CAD generates a complex ecological environment for novice students to operate within. Having to externalise a conceptual idea on a system where they may not have attained an appropriate level of associated knowledge while at the same time having to do so with procedural considerations can be problematic and therefore presents an unknown entity which students need to negotiate. Smith (2001) notes a limitation of modelling as being a lack of ability to learn how to create the model and this study aims to explore the strategies students employ when confronted with this situation. This study therefore aims to test a two part hypothesis. The first hypothesis is that students may satisfice their design, a concept described as determining "a path that will permit satisfaction at some specified level of all of its needs" 
(Simon 1956, p. 136) and that this will be achieved through the employment of fast and frugal heuristics. This paper describes a comparative study between two fundamentally different CAD systems. As the CAD system is the primary independent variable and due to the opposing nature inherent to these systems, the second part of the hypothesis is that the heuristics employed will be different between the control and experimental group as ecologically rational decisions are made. The employment of such heuristics, while supporting the student in solving proximal problems, may be circumventing their attainment of learning objectives concerned with developing problem solving skills and the acquisition of knowledge. Furthermore, observing the heuristics which are evidenced during modelling episodes can provide insight into how students can be supported in similar pedagogical activities.

\section{Method}

\section{Approach}

The aim of the study was to explore the approaches to problem solving employed by students when CAD modelling. A comparative study was designed between two CAD systems of different natures; a parametric CAD modelling system and a freeform moulding CAD system. Two distinctly different CAD systems were included to investigate the potential for specific heuristics to be unique to each type of system. A control and experimental group were formulated within a cohort of post-primary students for the purposes of this study with the control group utilising SolidWorks and the experimental group utilising CRE8. Both groups were administered an identical design brief for which they had to conceive an idea and model it using their designated CAD system. Following this, the students were asked to model a prescribed organic geometry using their designated CAD system to induce further problem solving episodes thus affording the potential for a greater insight into the heuristics exhibited by the students. All modelling was captured and analysed through a visual and verbal protocol analysis (Middleton 2008) and an adaptation of Spillane et al. (2012) 'Multidimensional Problem-Solving Codex' was used to code the data of the modelling organic geometry task.

\section{Participants}

The study cohort $(n=15)$ consisted of senior cycle design and technology students at post-primary level within the Irish education system. The cohort had a mean age of 15.53 years with a standard deviation of 0.52 years and consisted of 12 males and 3 females. This ratio of males to females is representative of the demographic at a national level (SEC 2015). The participants all had prior experience of SolidWorks which, in conjunction with prior experience of design and technology at junior cycle, suggested their appropriateness as participants for this study.

A control and experimental group were formed within the study cohort. Participants were designated to either the control or experimental group based on their performance in an assessment consisting of both geometric problem solving and parametric modelling tasks. The average result of the experimental group was $61.25 \%$ and the average of the control group was $59.23 \%$. The experimental group consisted of eight participants and the control group consisted of seven. 


\section{Design of instruments}

As CAD modelling at Irish post-primary level is typically operationalised through SolidWorks it was selected for inclusion in this study (e.g. SEC 2016). As SolidWorks is a parametric modelling system, it was decided to include a CAD tool where a different modelling strategy is inherent to the software. A freeform surface modelling system called CRE8 was selected because it is a 3D design and moulding program. A Novint Falcon is required in conjunction with CRE8. The device provides haptic feedback to the user allowing them to interact with their model through touch. Figure 1 depicts a participant modelling with CRE8 and the Novint Falcon to create an organic geometry. Modelling with CRE8 has similarities to a Boolean approach to modelling as it involves selecting a primary geometry such as a cube, cone or cylinder and deforming it into the required form. Additional geometries can be subsequently added as necessary relative to the initial geometry and manipulated through deformation as appropriate.

The design brief required the participants to conceive a design for a chair which they would model using their designated CAD system. This geometry was selected to ensure a sufficient degree of familiarity with the object while providing a divergent task whereby the CAD modelling period would be goal orientated. The intent of this task was to examine the participants' capacity to conceive and realise a design idea through CAD with an understanding that pertinent levels of technical competency may be an inhibitor.

Subsequent to this design activity the participants were required to model a prescribed organic geometry using the same designated CAD system as before. This activity was introduced because it was envisioned that during the previous design task participants could conceive an idea which was within their capacity to model and therefore not present any problematic activity, consequently preventing an investigation into the heuristics they would exhibit. While the geometry of the organic stimulus was a familiar geometry, not having any prior influence on its design suggested an increased potential for problems to occur during the modelling task.

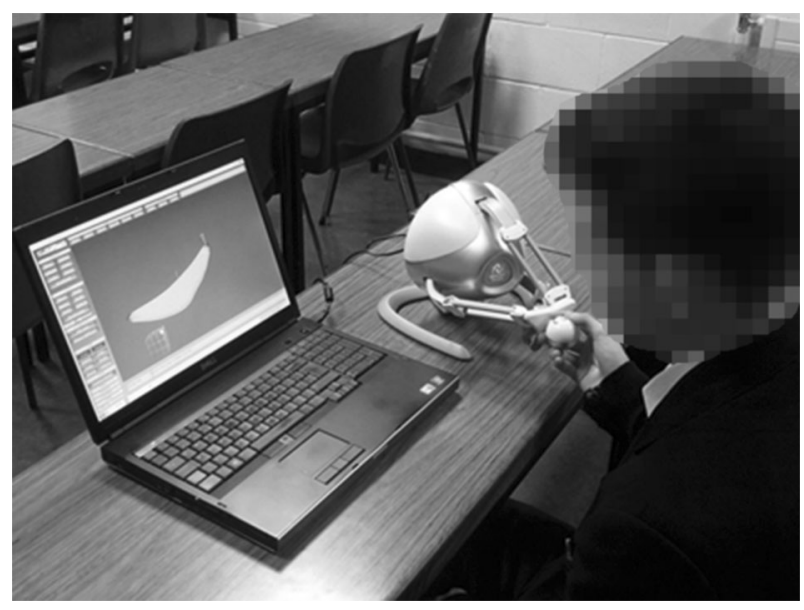

Fig. 1 A participant using CRE8 with the Novint Falcon to model an organic geometry 


\section{Implementation}

All participants had previous experience of SolidWorks having engaged with ten prescribed lessons prior to the study. These lessons were structured and focused on modelling primary solid geometries with a short period of time dedicated to unstructured exploration of the software. No participant had prior experience of CRE8 or the Novint Falcon prior to the study. A 20 min demonstration was delivered to the experimental group only. During this time the group received a short overview of the technology which illustrated each command within the software. This was followed by each participant experimenting with the software and hardware for one min while asking questions to clarify any queries as a group. They then participated in an individual modelling activity requiring them to model a geometry which they selected at their own discretion. This allowed participants to familiarise themselves with the software without being under observation by the remainder of the group. Each participant was allocated five min for this activity to allow for the development of personal constructs such as their own capability and the capacity of the modelling tool. Upon completion, the control and experimental group were introduced to the initial phase of the study which involved engaging with the design brief.

The design brief was administered to the participants as a group however they worked individually to conceptualise their ideas. All participants were afforded $30 \mathrm{~min}$ to generate a conceptual design under the premise that they would have to model it on their designated CAD system. A further 30 min was allocated for the formulation of a modelling strategy designed around Chester's (2007) 'CAD Workbook' as a strategy to facilitate the development of strategic knowledge pertinent to the modelling of their designs. Upon completion of their design, each participant was afforded 30 min individually to model their designs using the CAD system assigned to their group. Participants were permitted to request assistance while modelling during this activity to account for designs which were beyond the participants' level of capability. This assistance could only take the form of instruction concerning the use of specific features within the CAD systems and was delivered by a common member of the research team to ensure consistency. This experience was captured through both a visual and verbal protocol.

When all modelling was completed from the design activity, the second phase of CAD modelling was initiated. This was an individual activity whereby participants were asked to model a prescribed organic geometry within a five min time period. Again, the participants utilised the CAD system designated to their group and this activity was also captured using both a visual and verbal protocol. The time began immediately after a participant had received the instructions. No assistance was given during this activity. Upon one participants completion the next participant would begin. No interaction was permitted between participants throughout this phase.

\section{Treatment of data}

The video and verbal analyses were coded on a second by second basis to support a high level of accuracy. When coding the modelling activity from the initial design activity, it emerged that participants spent a relatively short amount of their time engaging with problems, with the majority of the time being spent progressively working through tasks. The control and experimental groups spent on average $20.09 \%$ and $4.98 \%$ of their time respectively engaging with problems, with problem solving episodes lasting as short as $4 \mathrm{~s}$ before the participants sought assistance. It was therefore decided to code their approaches 
to and actions within problems into six categories which were inductively derived from the data:

1. Persist To persist with a strategy without seeking additional information in an attempt to affect the outcome

2. Explore To persist with the same strategy but to seek other information in an attempt to positively affect the outcome

3. Alternative To adopt or search for an alternative strategy

4. Abandon To abandon the problem and focus on modelling a different element of the object

5. Seek assistance To ask for help in solving a problem

6. Assistance given The phase where the participant was receiving assistance

These phases were induced from an initial analysis of the data aiming to broadly categorise the type of behaviour exhibited during problem solving episodes with the analysis of the organic modelling activity focusing on the specific heuristics employed.

During the organic modelling activity the participants engaged with problems for longer periods of time without the option to request assistance with problems. It was therefore possible to gain a better insight into the heuristics which were employed. To analyse this dataset, the participants behaviour was coded onto Spillane et al.'s (2012) 'Multidimensional Problem-Solving Codex'. For the purposes of this study, the 'satisfycing' category was added to the original codex to account for cases where the exact intent of the behaviour was ambiguous but the action was implementary. The adapted codex which includes satisficing is presented below (Table 1).

Spillane et al.'s (2012) codex is inclusive of a number of heuristics as well as a number of behaviour descriptors. The heuristics included are:

- Recognition "If one of two objects is recognized and the other is not, then infer that the recognized object has the higher value with respect to the criterion" (Goldstein and Gigerenzer 2002, p. 76)

- Take the first "In familiar yet ill-defined tasks, choose one of the initial options generated once a goal (and strategy) has been defined, rather than exhaustively generating all possible options and subsequently processing them deliberatively" (Johnson and Raab 2003, p. 218)

- Working forwards "Proceeding from the given initial situation to the desired final situation, from the data to the unknown" (Polya 1957, p. 227)

- Working backwards "Start from what is required and assume what is sought is already found... inquire from what antecedent from the desired result could be derived" (Polya 1957, p. 227)

- Means-end analysis "The particular heuristic search system that finds differences between current and desired situations, finds an operator relevant to each difference, and applies the operator to reduce the difference" (Simon and Newell 1971, p. 152)

- Gaze "All relevant information is contained in one variable... attending to this one variable alone and ignoring all causal relevant information" (Raab and Gigerenzer 2005, p. 192)

- Generate and test "Each possible combination of the variables is systematically generated and then tested to see if it satisfies all the constraints. The first combination that satisfies all the constraints is the solution" (Kumar 1992, p. 33) 
Table 1 Problem-solving codex adopted in the study adapted from Spillane et al. (2012)

\begin{tabular}{|c|c|c|}
\hline Phase & No. & Descriptor/indicator \\
\hline \multirow[t]{7}{*}{ Identifying } & 1 & Recognition: Heuristic \\
\hline & 2 & Take the first: Heuristic \\
\hline & 3 & $\begin{array}{l}\text { Effort and energy put into establishing the given problem, constructing constraints, } \\
\text { identifying components }\end{array}$ \\
\hline & 4 & $\begin{array}{l}\text { Making sense of information in text, imagery, diagram, symbolism, signage, } \\
\text { checking components in task }\end{array}$ \\
\hline & 5 & Information is organised (verbally) \\
\hline & 6 & $\begin{array}{l}\text { Goals, parameters, constraints are represented by statements, pictorially (sketch, } \\
\text { signage, etc.) and verbally }\end{array}$ \\
\hline & 7 & Criteria/goals are established \\
\hline \multirow[t]{7}{*}{ Planning } & 8 & Working forwards: Heuristic \\
\hline & 9 & Working backwards: Heuristic \\
\hline & 10 & Concepts, knowledge and facts are assessed and considered \\
\hline & 11 & Various solutions approaches are considered \\
\hline & 12 & A conjecture/assumption is formulated \\
\hline & 13 & Strategic development of solution approach(es) is (are) imagined \\
\hline & 14 & Approach is determined \\
\hline \multirow[t]{8}{*}{ Implementing } & 15 & Means-ends analysis: Heuristic \\
\hline & 16 & Gaze: Heuristic \\
\hline & 17 & $\begin{array}{l}\text { Selection and implementation of various procedures (movement with purpose, plan } \\
\text { in action) }\end{array}$ \\
\hline & 18 & Constructs (logically/illogically) connected statements \\
\hline & 19 & Carries out set process/procedure/gives response (answer) \\
\hline & 20 & Satisficing \\
\hline & 21 & $\begin{array}{l}\text { Evidence of sense making/attempts to fit in new information with existing } \\
\text { schemata/plan/process }\end{array}$ \\
\hline & 22 & Validation of conjecture is considered \\
\hline \multirow[t]{5}{*}{ Evaluating } & 23 & Generate and test: Heuristic \\
\hline & 24 & Take the best: Heuristic \\
\hline & 25 & Results are tested for their suitability/reasonableness \\
\hline & 26 & Decision is made about validity of procedure/answer/solution \\
\hline & 27 & Cycles back or cycles forward based on results from checking/critiquing \\
\hline
\end{tabular}

- Take the best "The Take the Best algorithm assumes a subjective rank order of cues according to their validities...the highest ranking cue (that discriminates between the two alternatives) [is] the best cue" (Gigerenzer and Goldstein 1996, p. 653)

Many of the other behaviour descriptors stem from Polya's (1957) problem solving cycle which contains the four stages of 'understanding the problem', 'devising a plan', 'carrying out the plan' and 'looking back'. Bogard et al. (2013), when coding cognitive processes, also describe many of the processes described in this codex and were also to use this information to gain a significant insight into people's problem solving processes. 


\section{Findings}

\section{Initial modelling activity based on the design brief}

An overview of the results generated from analysing the participant's approaches to problem solving within the initial modelling activity is depicted in Table 2. A number of educationally relevant insights emerged from the results. The control group spent over twice as long on average ( $89.69 \mathrm{~s})$ engaging with the problems they encountered than the experimental group $(42.88 \mathrm{~s})$. This may be due to slight differences in the initial approaches to solving the problems evidenced by each group. The participants in the control groups initial strategy to solving 12 of the problems they encountered was to explore various ways to make their initial approach work while for 10 of the problems they immediately requested assistance. In comparison, only two of the problems encountered by the experimental group were initially explored while for nine problems assistance was immediately sought.

Another pertinent insight stems from the percentages of time each group spent during a problem engaging with certain approaches. The control group were more inclined to spend time persisting $(22.54 \%)$ with an approach and exploring (47.6\%) various strategies to getting that approach to work. The experimental group displayed a different strategy in that a significant portion of their problem solving episodes $(44.75 \%)$ involved receiving assistance. Additionally, assistance was sought at some stage during each problem encountered by the experimental group. Furthermore, no participant in either group attempted to identify an alternative approach to any problem they encountered.

Table 2 Overview of problem solving episodes from within the initial modelling activity

\begin{tabular}{lll}
\hline Statistic & Control group & Experimental group \\
\hline Modelling system & SolidWorks & CRE8 \\
Total no. of problems & 23 & 16 \\
Total no. of problems successfully resolved & 21 & 15 \\
Total no. of problems with assistance given & 18 & 16 \\
Average length of problem & $89.69 \mathrm{~s}$ & $42.88 \mathrm{~s}$ \\
Average length of problem (no assistance) & $114.4 \mathrm{~s}$ & N/A \\
Average length of problem (with assistance) & $82.93 \mathrm{~s}$ & $42.88 \mathrm{~s}$ \\
Average length of time receiving assistance & $28.47 \mathrm{~s}$ & $20.47 \mathrm{~s}$ \\
Average length of time before seeking assistance & $56.86 \mathrm{~s}$ & $40 \mathrm{~s}$ \\
Initial approach to problems & & \\
Explore & 12 & 2 \\
Persist & 1 & 5 \\
Seek Assistance & 10 & 9 \\
Percentage of time per approach & & 11.81 \\
Persist (\%) & 22.54 & 44.75 \\
Explore (\%) & 47.60 & 30.90 \\
Alternative (\%) & 0 & 0 \\
Seek assistance $(\%)$ & 6.40 & \\
Assistance given (\%) & 23.46 & \\
\hline
\end{tabular}


Both groups had similar success rates in resolving their problems. The control group had two unresolved problems. No assistance was sought in either problem. In both cases the participants abandoned the problems and progressed to modelling different aspects of their designs. The problem areas were not revisited with the features being omitted in the final models. There was one unresolved problem in the experimental group. During this problem the participant immediately sought assistance asking could flat geometry be created. The relevant feature was identified to the participant who decided not to include the feature. The problem areas were not revisited with the feature being omitted in the final model.

\section{Organic modelling activity}

Without the capacity to request assistance when modelling the organic geometries, a deeper insight could be generated into how the participants were approaching problems when CAD modelling with their respective software. The participants' behaviours were codified using the adapted version of Spillane et al.'s (2012) codex as previously discussed. Figure 2 illustrates an example of the results of generated through codifying behaviour using Spillane et al.'s (2012) codex. Utilising this codex can provide a more detailed insight into the behaviours and heuristics a person exhibits. For the purposes of this study it was decided to examine the typical heuristics which were evidenced by participants in this environment to initiate an investigation into the ecological rationality in their selection. It was also deemed appropriate to examine the amount of time participants spent in various stages of their problem solving cycles.

Both groups evidenced similar behaviour however there were some differences which may be attributional to the different modelling environments characteristic of the CAD systems and the resulting strategies they espouse. The control group who utilised SolidWorks typically carried out set processes (Kimbell 2011), generated and tested possible approaches (Linn and Petersen 1985), evidenced the gaze heuristic (Johnson and Raab 2003), made decisions about validity (Novick and Bassok 2005), determined an approach

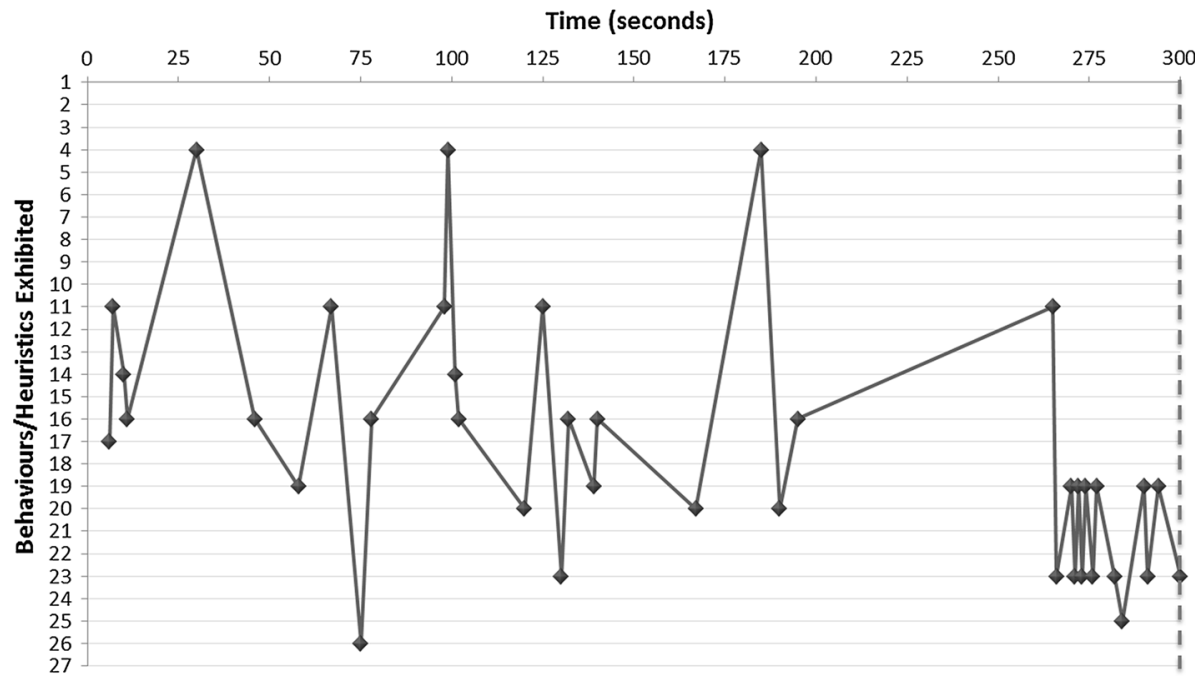

Fig. 2 Example behavioural analysis using the adapted version of Spillane et al.'s (2012) multidimensional problem solving codex 
(Goldstein and Gigerenzer 2002) and identifying the problem (Bhavnani et al. 1993). The experimental group who utilised CRE8 typically exhibited the gaze heuristic Johnson and Raab (2003), tested results (Miller 1956), made decisions about their validity (Novick and Bassok 2005) and to a lesser extend determined an approach (Goldstein and Gigerenzer 2002) and spent time making sense of information of perceived relevance to the problem (Bogard et al. 2013). The complete set of results from the analysis into the exhibition of heuristics and behaviours are depicted in Table 3.

Using this data, it was also of interest to analyse the time participants spent either identifying information, planning an approach, implementing an approach or evaluating an approach. The results of this analysis are presented in Fig. 3. In both groups the majority of time was spent implementing an approach which was following by evaluating, identifying critical elements and planning respectively to varying degrees.

\section{Discussion}

This study aimed to examine the heuristics and behaviours exhibited by students when creating CAD models utilising two different CAD systems. Stemming from this, it was hypothesised that when the students encountered a problem they would display heuristics to satisfice problems without identifying all necessary information. It addition to this, it was envisioned that these heuristics would be different between the control and experimental groups reflecting the differences in the CAD systems being used. The results of this study subsequently illustrated that while there was a difference in the individual heuristics and behaviours across both groups, generally all students behaved in a fundamentally similar way. At a macro level, the approaches to engaging in problems that were selected

Table 3 Average quantity per problem of behaviours and heuristics exhibited during the organic modelling activity

\begin{tabular}{|c|c|c|c|c|c|c|c|}
\hline \multicolumn{4}{|c|}{ Control group } & \multicolumn{4}{|c|}{ Experimental group } \\
\hline $\begin{array}{l}\text { Behaviour } \\
\text { code }\end{array}$ & $\begin{array}{l}\text { Average } \\
\text { quantity }\end{array}$ & $\begin{array}{l}\text { Behaviour } \\
\text { code }\end{array}$ & $\begin{array}{l}\text { Average } \\
\text { quantity }\end{array}$ & $\begin{array}{l}\text { Behaviour } \\
\text { code }\end{array}$ & $\begin{array}{l}\text { Average } \\
\text { quantity }\end{array}$ & $\begin{array}{l}\text { Behaviour } \\
\text { code }\end{array}$ & $\begin{array}{l}\text { Average } \\
\text { quantity }\end{array}$ \\
\hline 1 & 0.43 & 15 & 0.14 & 1 & - & 15 & - \\
\hline 2 & 0.57 & 16 & 5.57 & 2 & 0.63 & 16 & 7.63 \\
\hline 3 & 3.57 & 17 & 0.57 & 3 & 0.13 & 17 & 0.00 \\
\hline 4 & 3.00 & 18 & - & 4 & 2.13 & 18 & - \\
\hline 5 & - & 19 & 12.29 & 5 & - & 19 & 1.25 \\
\hline 6 & - & 20 & 1.71 & 6 & - & 20 & 0.25 \\
\hline 7 & - & 21 & 0.43 & 7 & 0.13 & 21 & - \\
\hline 8 & - & 22 & - & 8 & - & 22 & - \\
\hline 9 & - & 23 & 8.71 & 9 & - & 23 & 1.25 \\
\hline 10 & 0.14 & 24 & 2.86 & 10 & - & 24 & 0.75 \\
\hline 11 & 2.43 & 25 & 1.14 & 11 & 0.50 & 25 & 4.38 \\
\hline 12 & 0.14 & 26 & 4.29 & 12 & - & 26 & 3.38 \\
\hline 13 & 0.14 & 27 & - & 13 & - & 27 & - \\
\hline 14 & 3.71 & & & 14 & 2.25 & & \\
\hline
\end{tabular}


Control Group

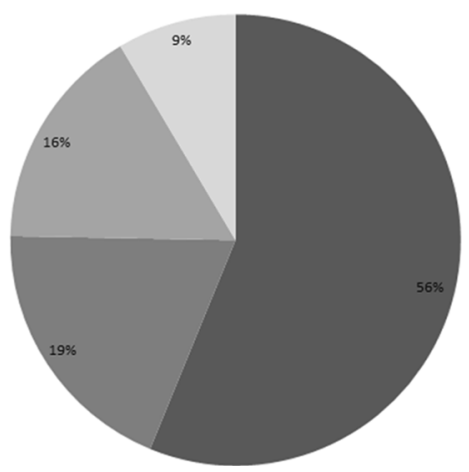

Experimental Group

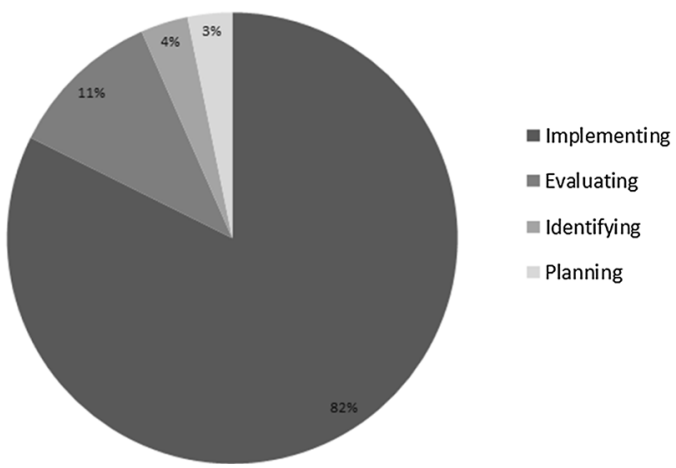

Fig. 3 Analysis of time (\%) spent in stages of problem solving cycles

suggest a circumvention of both learning processes and the engagement in activity which could lead to the acquisition of the strategic knowledge associated with CAD expertise, and the designerly knowledge inherent to the discipline. This has the potential to significantly impact student learning within this subject area as the philosophical underpinning of the discipline involves creating meaning through designerly activity. A misalignment in the synthesis between these two areas can prevent students from actively engaging in the learning process and ultimately prevent their attainment of pertinent educational goals.

This study generated a number of significant insights into the students' engagement with the problems they encountered while CAD modelling. An examination of a model can illustrate the physical procedures which resulted in its creation however the cognitive process which the student underwent is not necessarily apparent. From a learning perspective, this cognitive process is arguably the most beneficial aspect. While an efficient problem solving approach may be selected to optimise performance in a problem or educational experience, this efficiency has the potential to circumvent learning and therefore the future application of knowledge and skills may be hindered. Considering the factors suggested as being involved in the selection of a heuristic (Hutchinson and Gigerenzer 2005), it is possible to theorise the rationales underpinning the students decision making and problem solving processes. Perhaps the most interesting behaviour exhibited during the initial modelling task was the selection of strategies adopted by the students. For both groups, a significant portion of problems were solved by the student requesting assistance and in many cases this was the initial approach to the problem. With evolutionary training being cited as a factor in students determining such an approach, this may be suggestive of a pre-existing didactic contract the students were operating under. A didactic contract describes "the interplay of mutual expectations between teacher and students" (Verscheure and Amade-Escot 2007, p. 248). It may be the case that previous educational experiences created a problem solving culture defined by an understanding that people in the role of an educator will aid students in negotiating a problem through the provision of a solution. Such a culture could have removed the need for students to explore alternative approaches to encountered problems because they can solve it more efficiently by requesting assistance and dissolving their own responsibility as a learner. The significance of a problem lies in the relationship the learner has with the problem (Geiger and Galbraith 1998) and therefore the presence of a teacher may be circumventing the students 
forming that relationship as deep engagement is not an expectation. As the problems encountered were CAD modelling problems, the decision not to explore alternative approaches hinders the acquisition of strategic CAD knowledge. Therefore while the potential proximal effect of this heuristic is that problems are successfully solved, the distal effects may be that learning goals pertinent of the development of problem solving competencies and strategic knowledge aren't being reached. This is not to suggest that teachers should remove themselves entirely from the problem solving equation. Vygotsky's (1978) sociocultural theory describes learning as a social process occurring on two levels, "first on the social level, and later, on the individual level; first, between people (interpsychological) and then inside the child [learner] (intrapsychological)" (Vygotsky 1978, p. 57). This theory requires teachers to become co-constructers of learning with their students. As a co-constructor of knowledge, the role of the teacher should concern the guidance and facilitation of the student in reaching a solution rather than being a provider of solutions.

An examination of the time spent in each stage of a problem solving cycle by the students during the organic modelling task reveals a significantly low proportion of time being spent planning an approach. The majority of time was spent in an implementation stage followed by an evaluation stage. It is posited that additional time being afforded to planning and identifying critical information may have supported the learners more in these activities to develop a higher degree of flexibility in their capacity to engage with these and future problems. "Flexible problem solving is associated with the ability to apply one's knowledge structures in relatively new situations" (Kalyuga and Hanham 2011, p. 63) and there are a number of frameworks to support the development of such problem solving skills. These include the "Theory of Inventive Problem Solving Technique" (Akay et al. 2008) and "Systematic Inventive Thinking" (Barak and Goffer 2002) among others. Both of these frameworks have potential within an educational context (Seery and Delahunty 2015). However considering these students as novices, and based on the behaviours they exhibited, it may be more beneficial to design pedagogical strategies for people at this stage of their education which inform them about problem solving cycles. Combined with a strategy which aims to develop the core knowledge base of the pertinent discipline, this approach may aid in alleviating the reoccurrence of problems of a similar nature in future activities supporting students in a deeper exploration of the subject.

An examination into the behaviours and heuristics most commonly demonstrated by the students supports the evidence suggesting a lack of planning while engaging with a problem. While implementing an approach, the gaze heuristic, describing a focus on a single variable of information and ignoring causal information, was regularly displayed by both groups. Other regularly evidenced behaviours provide insight into how an approach was determined. In both groups the students typically would not plan an approach but rather select an approach and test its validity. If the approach proved to be unsuccessful, another would be selected and tested. This again suggests a need to teach students to plan their approaches before implementation and try to envisage potential outcomes.

In response to the hypotheses underpinning this study, the findings suggest that the participants in both groups did satisfice their designs. It is theorised that this is due to the combination of a previously developed didactic contract and limitations in their cognitive capacities. The exact weight of the influence each of these aspects has is undeterminable from the results of this study, however further work to elicit this would be a significant contribution to the discipline. With respect to the hypothesis that participants would utilise different heuristics as a result of the modelling tool allocated to their group, the findings of this study illustrate that this needs to be considered at two levels. At a macro level, the 
nature of the heuristics adopted by both groups were similar with the predominant heuristics selected concerning implementation, followed by evaluation, identification and finally planning. However, at a micro level, the exact heuristics used differed within these categories. The findings from the macro level categories can have a direct impact within education as support can be given to students in the planning stages of problem solving. However further work is needed to examine the exact effects on learning that individual heuristics can have. The high occurrence of the gaze heuristic suggests that from a pedagogical perspective, engaging students in reflective practices concerning the outcomes of their approaches may aid in their problem solving success and the learning they can achieve from problem engagement. Ultimately, an amalgamation of the evidence presented in this paper illustrates the students aligning with the behavioural characteristics of novices in that their overarching approach was that of a means-end heuristic (Novick and Bassok 2005). The behaviours they exhibited demonstrate both an underdeveloped knowledge base and approaches which, while potentially optimal in the completion of the activities themselves, are suboptimal in the development and acquisition of further pertinent knowledge.

While the results of this study offer significant educational insight, further research is needed into the behaviours exhibited by students and their subsequent effects during educational transactions. The study cohort, while representative of the demographic of technology education at a national level, contained only three female students. Linn and Petersen (1985) provide evidence showing that males and females engage with problems in different ways. Their results identify females as more analytical and males as more holistic. Therefore, further work should consider the potential for gender differences in the adoption of heuristics in educational settings. In addition, while in this study the nature of the students' previous educational experience likely influenced their behaviour, this past experience was uncontrolled. It would be of interest to examine behaviours from a longitudinal perspective where previous educational experiences could be designed to examine the effect of such experiences on behaviour selection. Finally, it is important to note that the use of heuristics can be both positive and negative. Students can adopt heuristics to alleviate some of the cognitive load being experienced during a learning activity resulting in an increased capacity for learning. In design activities, the use of heuristics can also result in an optimal solution within the constraints of the problem. However, a negative implication can be that exposure to critical information may be circumvented. It is therefore critical that educators are conscious of the potential for this to occur and that they ensure the elements of the learning activity they determine to be essential are clearly expressed to students.

Open Access This article is distributed under the terms of the Creative Commons Attribution 4.0 International License (http://creativecommons.org/licenses/by/4.0/), which permits unrestricted use, distribution, and reproduction in any medium, provided you give appropriate credit to the original author(s) and the source, provide a link to the Creative Commons license, and indicate if changes were made.

\section{References}

Akay, D., Demiray, A., \& Kurt, M. (2008). Collaborative tool for solving human factors problems in the manufacturing environment: the theory of inventive problem solving technique (TRIZ) method. International Journal of Production Research, 46(11), 2913-2925. 
Barak, M., \& Goffer, N. (2002). Fostering systematic innovative thinking and problem solving: lessons education can learn from industry. International Journal of Technology and Design Education, 12(3), 227-247.

Bhavnani, S., Garrett, J., Jr., \& Shaw, D. (1993). Leading indicators of CAD experience. In U. Flemming \& S. van Wyk (Eds.), 5th international conference on computer-aided architectural design futures (pp. 313-334). Pittsburgh: Elsevier Science Publishers.

Bogard, T., Liu, M., \& Chiang, Y. V. (2013). Thresholds of knowledge development in complex problem solving: A multiple-case study of advanced learners' cognitive processes. Educational Technology Research and Development, 61(3), 465-503.

Chase, W., \& Simon, H. (1973). Perception in chess. Cognitive Psychology, 4(1), 55-81.

Chester, I. (2007). Teaching for CAD expertise. International Journal of Technology and Design Education, $17(1), 23-35$.

Cowan, N. (2001). The magical number 4 in short-term memory: A reconsideration of mental storage capacity. Behavioral and Brain Sciences, 24(1), 87-114.

Galton, F. (1880). Statistics of mental imagery. Mind, 5(19), 301-318.

Geiger, V., \& Galbraith, P. (1998). Developing a diagnostic framework for evaluating student approaches to applied mathematics problems. International Journal of Mathematics, Education, Science and Technology, 29(4), 533-559.

Gigerenzer, G. (2001). The adaptive toolbox. In G. Gigerenzer \& R. Selten (Eds.), Bounded rationality: The adaptive toolbox (pp. 37-50). Cambridge, Massachusetts: The MIT Press.

Gigerenzer, G. (2004). Fast and frugal heuristics: The tools of bounded rationality. In D. Koehler \& N. Harvey (Eds.), Blackwell handbook of judgment and decision making (pp. 62-88). Oxford, UK: Blackwell.

Gigerenzer, G. (2008). Why heuristics work. Perspectives on Psychological Science, 3(1), $20-29$.

Gigerenzer, G., \& Goldstein, D. (1996). Reasoning the fast and frugal way: Models of bounded rationality. Psychological Review, 103(4), 650-669.

Goldstein, D., \& Gigerenzer, G. (2002). Models of ecological rationality: The recognition heuristic. Psychological Review, 109(1), 75-90.

Hutchinson, J., \& Gigerenzer, G. (2005). Simple heuristics and rules of thumb: Where psychologists and behavioural biologists might meet. Behavioural Processes, 69(2), 97-124.

Johnson, J., \& Raab, M. (2003). Take the first: Option-generation and resulting choices. Organizational Behavior and Human Decision Processes, 91(2), 215-229.

Kalyuga, S., \& Hanham, J. (2011). Instructing in generalized knowledge structures to develop flexible problem solving skills. Computers in Human Behavior, 27(1), 63-68.

Kaufman, J., Baer, J., Cropley, D., Reiter-Palmon, R., \& Sinnett, S. (2013). Furious activity vs. understanding: How much expertise is needed to evaluate creative work? Psychology of Aesthetics, Creativity, and the Arts, 7(4), 332-340.

Kimbell, R. (2011). Wrong... but right enough. Design and Technology Education: An International Journal, 16(2), 6-7.

Kirschner, P., Sweller, J., \& Clark, R. (2006). Why minimal guidance during instruction does not work: An analysis of the failure of constructivist, discovery, problem-based, experiential, and inquiry-based teaching. Educational Psychologist, 41(2), 75-86.

Kumar, V. (1992). Algorithms for constraint satisfaction problems: A survey. AI Magazine, 13(1), 32-44.

Lang, G., Eberts, R., Gabel, M., \& Barash, M. (1991). Extracting and using procedural knowledge in a CAD task. IEEE Transactions on Engineering Management, 38(3), 257-268.

Linn, M., \& Petersen, A. (1985). Emergence and characterization of sex differences in spatial ability: A meta-analysis. Child Development, 56(6), 1479-1498.

Middleton, H. (2008). Examining design thinking: visual and verbal protocol analysis. In H. Middleton (Ed.), Researching technology education: methods and techniques (pp. 193-212). Rotterdam: Sense Publishers.

Miller, G. (1956). The magical number seven, plus or minus two: Some limits on our capacity for processing information. Psychological Review, 63(2), 81-97.

Novick, L., \& Bassok, M. (2005). Problem Solving. In K. Holyoak \& R. Morrison (Eds.), The Cambridge handbook of thinking and reasoning (pp. 321-349). New York: Cambridge University Press.

Payne, J., Bettman, J., \& Johnson, E. (1993). The adaptive decision maker. Cambridge, Massachusetts: Cambridge University Press.

Piaget, J. (1970). Piaget's theory. In P. H. Mussen (Ed.), Carmichael's manual of child psychology (3rd ed., pp. 703-732). New York: Wiley.

Polya, G. (1957). How to solve it: A new aspect of mathematical method (2nd ed.). Princeton, New Jersey: Princeton University Press. 
Raab, M., \& Gigerenzer, G. (2005). Intelligence as smart heuristics. In R. Sternberg \& J. Pretz (Eds.), Cognition and intelligence (pp. 188-207). Cambridge: Cambridge University Press.

Rodriguez, J., Ridge, J., Dickinson, A., \& Whitwam, R. (1998). CAD training using interactive computer sessions. In 105th ASEE annual conference \& exposition. Seattle, Washington.

Rynne, A., Gaughran, W., \& McNamara, B. (2003). Parametric modelling training strategies to capture design intent. In The 17th International Conference on Production Research (pp. 1-8). Blacksburgh, Virginia: International Foundation of Production Research.

Sargent, T. (1993). Bounded rationality in macroeconomics. Oxford, UK: Oxford University Press.

Schneider, J., \& McGrew, K. (2012). The cattell-horn-carroll model of intelligence. In D. Flanagan \& P. Harrison (Eds.), Contemporary intellectual assessment: theories, tests, and issues (3rd ed., pp. 99-144). New York: Guilford Press.

Schoenfeld, A. (1983). The wild, wild, wild, wild, wild world of problem solving (A review of sorts). For the Learning of Mathematics, 3(3), 40-47.

Schraw, G., Dunkle, M., \& Bendixen, L. (1995). Cognitive processes in well-defined and ill-defined problem solving. Applied Cognitive Psychology, 9(6), 523-538.

Schütze, M., Sachse, P., \& Römer, A. (2003). Support value of sketching in the design process. Research in Engineering Design, 14(2), 89-97.

SEC. (2015). Design and communication graphics: Chief examiners report. Dublin: State Examinations Commission.

SEC. (2016). Design and communication graphics: Student assignment. M80P/M81P, Dublin: State Examinations Commission.

Seery, N., \& Delahunty, T. (2015). Cognitive load as a key element of instructional design and its implications for initial technology teacher education. In M. Chatoney (Ed.), PATT29 (pp. 364-372). Palais du Pharo, Marseille: PATT.

Shah, A., \& Oppenheimer, D. (2008). Heuristics made easy: An effort-reduction framework. Psychological Bulletin, 134(2), 207-222.

Simon, H. (1955). A behavioral model of rational choice. The Quarterly Journal of Ecomnomics, 69(1), 99-118.

Simon, H. (1956). Rational choice and the structure of the environment. Psychological Review, 63(2), 129-138.

Simon, H., \& Newell, A. (1971). Human problem solving: The state of the theory in 1970. American Psychologist, 26(2), 145-160.

Smith, J. (2001). The DATA lecture: The current and future role of modelling in design and technology. The Journal of Design and Technology Education, 6(1), 5-15.

Spearman, C. (1904). "General Intelligence", Objectively Determined and Measured. The American Journal of Psychology, 15(2), 201-292.

Spillane, J., Seery, N., Canty, D., \& Lane, D. (2012). Observational study of students' individual heuristics when solving technological problems. In 119th ASEE annual conference \& exposition. San Antonio, Texas: American Society for Engineering Education.

Sternberg, R., Grigorenko, E., \& Bundy, D. (2001). The predictive value of IQ. Merrill-Palmer Quarterly, 47(1), 1-41.

Sweller, J. (1988). Cognitive load during problem solving: Effects on learning. Cognitive Science, 12(2), 257-285.

Verscheure, I., \& Amade-Escot, C. (2007). The gendered construction of physical education content as the result of the differentiated didactic contract. Physical Education and Sport Pedagogy, 12(3), 245-272.

Vygotsky, L. (1978). Mind in society: The development of higher psychological processes. Cambridge: Harvard University Press.

Williams, P. J., Iglesias, J., \& Barak, M. (2008). Problem based learning: Application to technology education in three countries. International Journal of Technology and Design Education, 18(4), 319-335. 